\title{
Hemofilia B o enfermedad de Christmas
}

\section{Hemophilia B or Christmas disease}

\author{
María A. Acosta-Aragón' ${ }^{1}$, Angélica R. Álvarez-Mina², \\ Julián C. Velásquez-Paz ${ }^{3}$, Jennifer C. Vizcaíno-Carruyo ${ }^{4}$
}

Resumen. La hemofilia B o enfermedad de Christmas se diferenció por primera vez de la hemofilia A en 1947. Su forma clásica consiste en un trastorno hereditario de la coagulación causado por mutaciones en el gen F9, que codifica para el factor IX de la coagulación. Su herencia está ligada al cromosoma $X$; las mujeres son portadoras, pero se manifiesta clínicamente en hombres, aunque se han descrito casos de mujeres portadoras sintomáticas. El factor IX activado es una proteína dependiente de vitamina $K$, sintetizada en el hígado, que forma parte del complejo tenasa, cuya función es formar la mayor cantidad de trombina en el nuevo modelo de la coagulación basado en células. De acuerdo a la actividad del factor IX, su deficiencia se puede clasificar en leve (5\% a $40 \%)$, moderada ( $1 \%$ a $5 \%$ ), o severa $(<1 \%)$. Su diagnóstico se realiza con la presencia de un TPT alargado que corrige con plasma normal y con la determinación del nivel funcional del factor IX, y se confirma con el estudio molecular que demuestra la mutación en el gen F9. Su diagnóstico diferencial incluye otras patologías como la hemofilia A. El tratamiento con factor IX recombinante es el más utilizado en la actualidad, pero se vienen desarrollando nuevas terapias con virus adeno-asociados recombinantes que prometen mejorar la calidad de vida para algunos pacientes afectados. La profilaxis juega un papel fundamental, en particular en los casos de enfermedad moderada y severa.

Palabras clave: hemofilia B, enfermedad de Christmas, factor IX, tiempo de tromboplastina parcial, cromosoma $X$.

\footnotetext{
${ }^{1}$ Médica, MSc en Biología-Genética Clínica, PhD en Genética de Poblaciones Humanas y Genética Forense. Profesora Titular, Departamento de Pediatría, Facultad de Ciencias de la Salud, Universidad del Cauca. Popayán, Colombia. E-mail: morin1924@gmail.com.

${ }^{2}$ Médica General, Consulta Externa, IPS Vivir, EPS Sura. Cali, Colombia.

${ }^{3}$ Médico General. Profesor de Semiología, Hospital Universitario San José de Popayán, Universidad del Cauca. Popayán, Colombia.

${ }^{4}$ Médica, Especialista en Hematología. Asistente Científica, Editora Médica Colombiana S.A. Medellín, Colombia.

Conflicto de interés: los autores declaran que no tienen conflicto de interés.

Medicina \& Laboratorio 2020;24:273-289. https://doi.org/10.36384/01232576.336

Recibido el 25 de abril de 2020; aceptado el 2 de julio de 2020. Editora Médica Colombiana S. A., $2020^{\circ}$.
} 
Abstract. Hemophilia B or Christmas disease was first differentiated from hemophilia $A$ in 1947. Its classic form consists of an inherited bleeding disorder caused by mutations in the F9 gene, which codes for coagulation factor IX. Its inheritance is linked to the $X$ chromosome; women are carriers, but it manifests clinically in men, although cases of symptomatic women carriers have been described. Factor $I X$ activates a vitamin K-dependent protein, synthesized in the liver, which is part of the tenase complex whose function is to form the largest amount of thrombin (factor Ila) in the new model of cell-based coagulation. According to factor IX activity, its deficiency can be classified as mild (5\% to 40\%), moderate (1\% to 5\%), and severe $(<1 \%)$. The diagnosis is made when there is a prolonged TPT that corrects with normal plasma, and by assessing the functional level of factor IX. The diagnosis is confirmed by molecular analysis that demonstrates the F9 gene mutation. Its differential diagnosis includes disorders such as hemophilia A. Treatment with recombinant factor $I X$ is widely used, but also new therapies are being developed with recombinant adeno-associated viruses that promise to improve the quality of life for some of these patients. Prophylaxis plays an important role in cases of moderate and severe disease.

Keywords: hemophilia B, Christmas disease, factor IX, partial thromboplastin time, $X$ chromosome.

\section{Introducción}

La hemofilia tipo B o enfermedad de Christmas, llamada así en honor al primer paciente descrito con esta enfermedad, se diferenció de la hemofilia $A$ en 1947, cuando el Dr. Alfredo Pavlovsky, hematólogo argentino, hizo la demostración de la corrección mutua del defecto al mezclar los plasmas de dos pacientes con diagnóstico de hemofilia A y B [1]. La forma clásica de la hemofilia B consiste en un trastorno hereditario de la coagulación causado por déficit del factor IX, el cual está codificado por el gen F9 [2]. Ocurre con una prevalencia de 1 en 30.000 y afecta a todas las razas $[3,4]$; además, es cinco a seis veces menos común que la hemofilia clásica tipo A [3].

La hemofilia $B$ es una enfermedad recesiva ligada al cromosoma $X[5,6]$, de carácter hereditario, y en raras ocasiones de aparición esporádica asociada a enfermedades autoinmunes o neoplasias [7]. Debido a su patrón de herencia recesivo ligado al $X$, se manifiesta clínicamente en los hombres, en tanto que las mujeres son las portadoras, aunque la pueden padecer bajo condiciones muy especiales [5,8]; entre ellas, si ambos padres tienen el gen afectado, en caso de inactivación del cromosoma $X$ no afectado durante la embriogénesis, o en pacientes con síndrome de Turner portadoras del gen mutado [9]. El patrón de herencia recesivo ligado al $X$ le confiere el $50 \%$ de probabilidad de padecer la enfermedad a cada nuevo hijo de sexo masculino, en tanto que el $50 \%$ de las hijas serían portadoras (figura 1).

\section{Estructura y expresión del gen $F 9$}

El precursor del factor IX es sintetizado en el hígado y circula en el plasma como zimógeno; una molécula inactiva formada por una cadena polipeptídica 


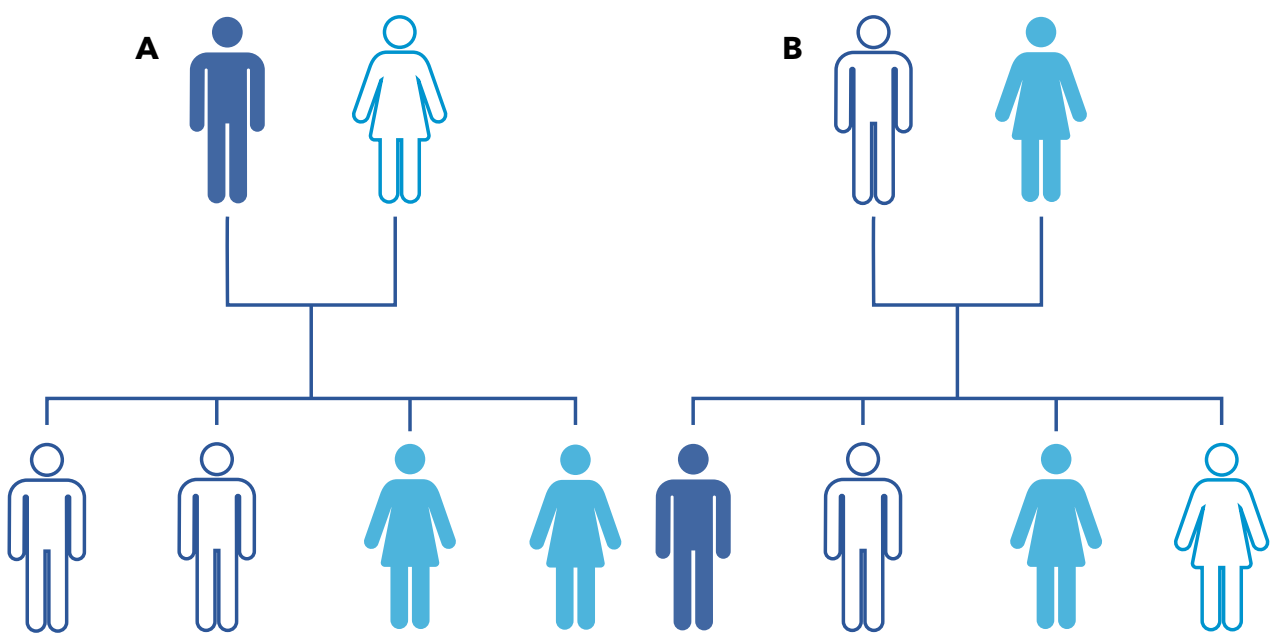

Figura 1. Transmisión genética de la hemofilia B. En azul oscuro, los pacientes con hemofilia, y en azul claro, las portadoras.

de 415 aminoácidos y 57 kDa, con una vida media en circulación de 24 horas aproximadamente [10-12]. El gen que codifica para este factor es el F9, localizado en el brazo largo del cromosoma $X$ en la posición q26.3-27.1 [13]. Tiene 8 exones que transcriben un ARNm de $2,8 \mathrm{~Kb}$, y de este, $1,4 \mathrm{~Kb}$ traducen una proteína precursora de 461 aminoácidos [14]. El precursor del factor IX posee varios dominios, incluyendo un dominio Gla (12 residuos de ácido glutámico), dominios EGF-1 (EGF, del inglés, Epidermal Growth Factor) y EGF-2, un péptido de activación y un dominio de serina proteasa. Mediante clivajes proteolíticos se remueven el péptido de señalización y los dominios del propéptido, y de esta forma el precursor se convierte en zimógeno del factor IX. Posteriormente, el zimógeno se libera a la circulación sanguínea, donde por acción del factor XI activado (Xla) o del complejo factor tisular/factor VII se cliva el péptido de activación, lo cual conduce a la conversión del factor IX a factor IXa. El factor IXa está compues- to por una cadena liviana (Gla, EGF-1, EGF-2) y una cadena pesada (dominio serina proteasa) unidos por un puente disulfuro [15-18] (figura 2).

Las mutaciones en el gen F9 impiden la producción de factor IX, lo cual conduce a los síntomas asociados con la hemofilia $[19,20]$. Se ha encontrado gran variabilidad en las mutaciones del gen F9, incluyendo mutaciones puntuales, deleciones, inserciones, cambios complejos y polimorfismos neutrales, entre otros defectos. En el $65 \%$ de los casos de hemofilia B, las mutaciones con cambio de sentido son las más frecuentes $[4,5,21,22]$. Las mutaciones puntuales simples en la región promotora del factor IX se correlacionan con la hemofilia B Leyden, en la cual los individuos que la padecen tienen una alteración en la producción del factor IX asociado a una enfermedad hemorrágica severa en la infancia, que desaparece después de la pubertad o posterior a la administración de testosterona [13,15]. Algunos estudios 


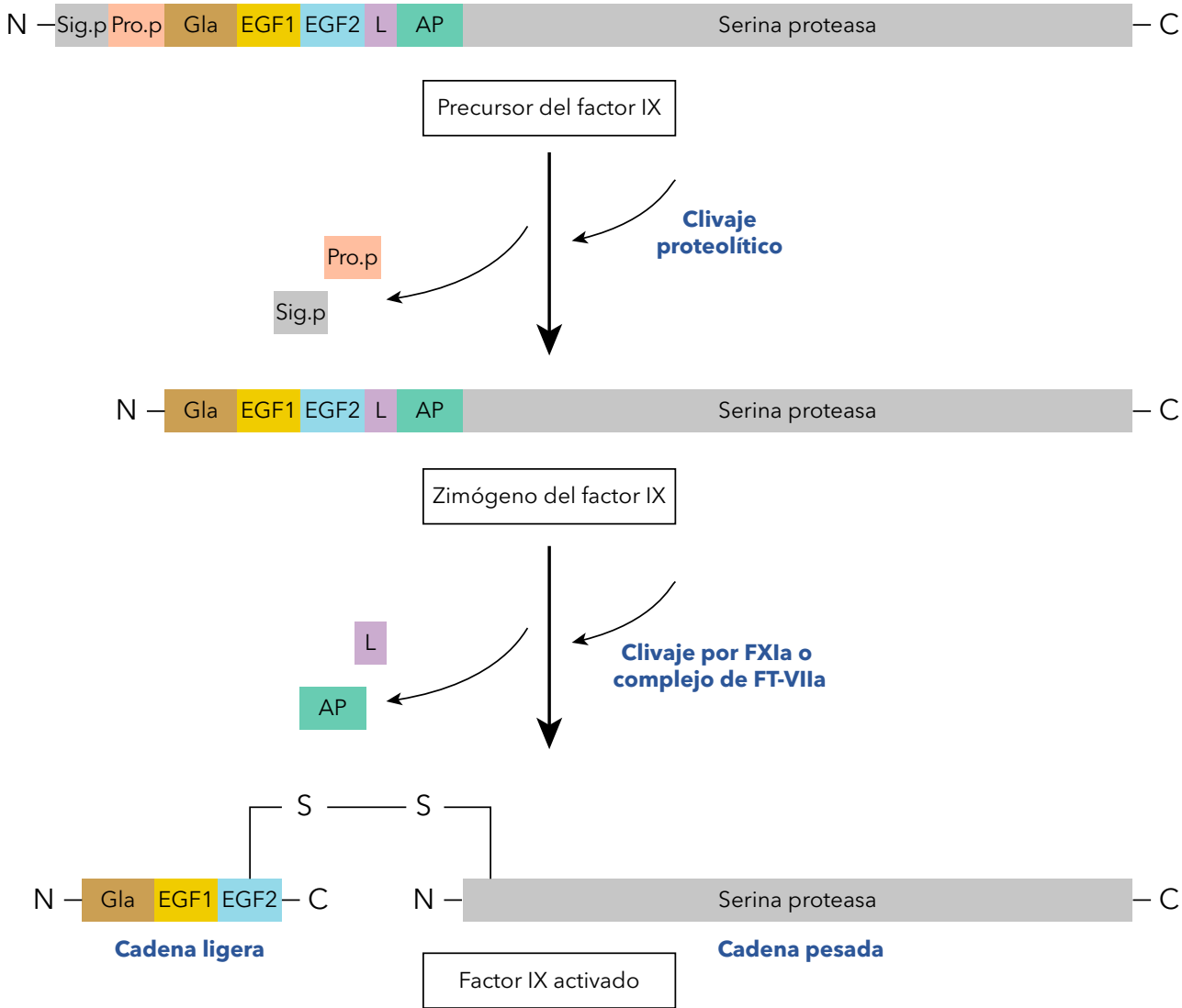

Figura 2. Estructura del factor IX. Sig.p: péptido de señalización; Pro.p: propéptido; Gla: dominio con residuos de ácido glutámico; EGF1 y 2: dominios del factor de crecimiento epidérmico 1 y 2; L: enlazador; AP: péptido de activación.

experimentales demuestran que la unión del receptor de andrógenos a la región promotora del factor IX regula la expresión del mismo [13].

\section{Coagulación}

El factor IX es un componente importante de la coagulación, y a pesar de que el modelo de la cascada clásica de la coagulación ha sido actualizado durante las últimas décadas, todavía mostraba deficiencias para explicar el proceso de la hemostasia in vivo [23]. Esto motivó el desarrollo de un nuevo modelo de coagulación, que además de incorporar el papel vital de las células, corrige las deficiencias del modelo previo. Para tener una visión más clara de la fisiopatología y clínica de la hemofilia $B$, se hará una descripción de la cascada clásica de la coagulación, y adicionalmente, se presentará el nuevo modelo celular de la coagulación.

\section{Cascada clásica de la coagulación}

Los eventos de coagulación no son iguales in vivo e in vitro [18]. In vitro, la activación de la cascada de la coagu- 
lación se inicia por la presencia de las cargas negativas del vidrio del tubo de recolección de la muestra, que activan el factor XII de la vía intrínseca, el cual convierte precalicreína (unida al cininógeno de alto peso molecular, QAPM) a calicreína, la cual, por retroalimentación positiva, activa más factor XII. El factor XIla activa al factor $\mathrm{XI}$, y este, a su vez, participa en la activación del factor IX. El factor IXa con ayuda del cofactor VIIla, calcio y fosfolípidos (complejo tenasa) (figura 3), es el encargado de transformar el factor $\mathrm{X}$ a su forma activa; por otro lado, la vía extrínseca se activa por la presencia de factor tisular (FT) o tromboplastina tisular, que activa al factor VII formando otro complejo que también participa en la activación del factor $X$ (vía común), quien junto con su cofactor $\mathrm{Va}$, calcio y fosfolípidos (complejo protrombinasa), convierte protrombina en trombina (factor Ila), la cual facilita la transformación del fribrinógeno en fibrina, una proteína filamentosa e insoluble que tiene la capacidad de formar redes y coágulos blandos con ayuda del factor estabilizador de la fibrina (XIII), para frenar el sangrado $[11,24]$.

\section{Nuevo modelo celular de la coagulación}

El nuevo modelo de la coagulación surge de la necesidad para explicar la ausencia de manifestaciones clínicas en las deficiencias de algunos factores

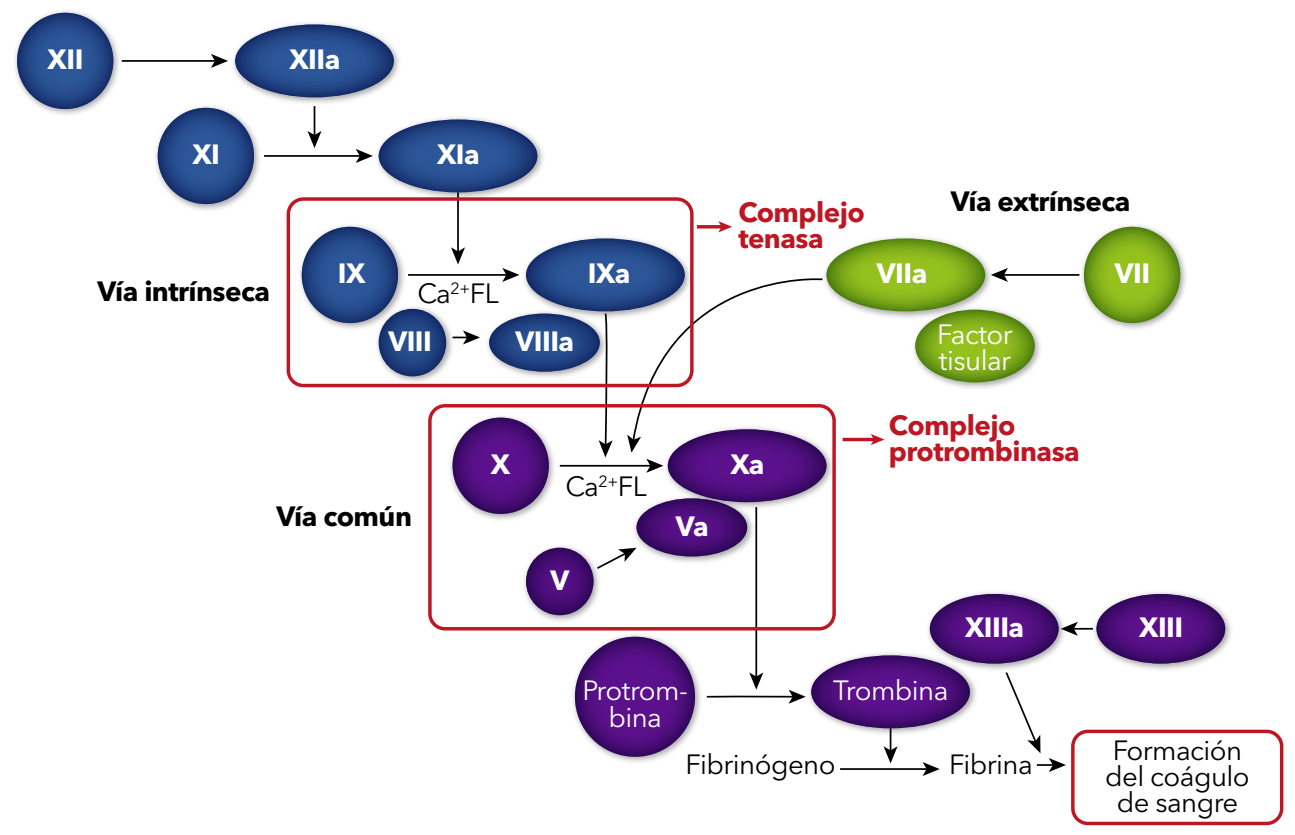

Figura 3. Cascada clásica de la coagulación. In vitro, el complejo FXII/precalicreína/quininógeno de alto peso molecular (QAPM) inicia la activación de la cascada, el cual activa al factor XI, que a su vez, activa el factor IX. Este, junto con su cofactor VIIla, $\mathrm{Ca}^{2+}$ y fosfolípidos (FL) (complejo tenasa), activan al factor $\mathrm{X}$; por otro lado, el factor tisular activa al factor VII, formando un complejo que activa también al factor $X$ (vía común), el que a su vez junto a su cofactor $\mathrm{Va}, \mathrm{Ca}^{2+}$ y $\mathrm{FL}$ (complejo protrombinasa), contribuyen a la formación de trombina (factor lla), y finalmente al coágulo de fibrina. 
como el XII, precalicreína y cininógeno, o la presencia de manifestaciones hemorrágicas importantes en otras (la de los factores $\mathrm{XI}, \mathrm{X}$ y $\mathrm{VIII}$ ). Al incorporar células como las plaquetas, explica adecuadamente la importancia de la interacción de ellas con los factores de coagulación para la generación del factor $\mathrm{Xa}$, y posteriormente de trombina. Además, este modelo permite una mejor comprensión del proceso hemostático in vivo, para realizar un buen diagnóstico y un manejo adecuado de los trastornos de la hemostasia [23].

En el modelo celular de la coagulación queda clara la importancia de los factores VIII y IX, ya que estos cumplen un papel crítico en la producción sostenida de trombina, y su ausencia conduce a trastornos hemorrágicos importantes. En este nuevo enfoque de la coagulación se identificó que no existen dos vías independientes de activación (intrínseca y extrínseca), sino que ambas vías están interconectadas y suceden de forma simultánea. El complejo FT/ VIla (de la vía extrínseca) tiene un papel principal en la nueva cascada, ya que activa al factor IX $y$ al factor $X$ al mismo tiempo, y es el que inicia la coagulación; un concepto diferente a lo descrito en la vía intrínseca, al demostrarse que la deficiencia grave del complejo formado por factor XII, precalicreína y cininógeno de alto peso molecular, no causaba trastornos hemorrágicos $[11,25]$. Este nuevo modelo le da importancia en la coagulación in vivo a la función de las superficies celulares (plaquetas, monocitos, músculo liso y células endoteliales), ya que exponen los fosfolípidos de membrana, que junto con el calcio, activan los complejos procoagulantes.

En el modelo celular de la coagulación se describen 3 fases: la fase de iniciación, en donde el complejo FT/
VIla/Ca ${ }^{2+} / \mathrm{FL}$ activa al factor IX y al factor $X$ para formar solo pequeñas cantidades de trombina, situación que se asocia también al hecho de que el factor Xa se inhibe por el inhibidor de la vía del factor tisular (TFPI, del inglés, Tissue Factor Pathway Inhibitor) [25]; la fase de amplificación, en donde la trombina formada activa a los factores XI, VIII y V, los cuales funcionan como cofactores y estimulan a su vez mayor formación de trombina, la cual acelera la activación plaquetaria y favorece la unión de los factores de coagulación a las plaquetas; $y$, la fase de propagación, en donde el factor Xla activa el factor IX, el cual en forma de complejo tenasa (IXa/VIIIa/Ca ${ }^{2+} / \mathrm{FL}$ ) participa en la activación de grandes cantidades de factor $X$, que junto con su cofactor $\mathrm{Va} / \mathrm{Ca}^{2+} / \mathrm{FL}$ (complejo protrombinasa), convierte la protrombina en trombina, y esta, el fibrinógeno en fibrina [13,24,26] (figura 4).

\section{Manifestaciones clínicas}

Los niveles normales de actividad de coagulación del factor IX en plasma se pueden expresar en porcentajes o en unidades internacionales (UI), que es la cantidad de actividad del factor IX por $\mathrm{mL}$ de plasma normal. Su concentración fisiológica es de 0,50 UI/mL a 1,50 UI/ $\mathrm{mL}$, o de $50 \%$ a $150 \%$ [27]. Los niveles por debajo de $50 \%$ determinan la sintomatología del paciente, que se caracteriza por una tendencia hemorrágica proporcional al grado de deficiencia del factor hemostático, aunque suele haber excepciones. En los casos graves la hemorragia ocurre de forma espontánea y repetitiva, usualmente como hemartrosis con daño a las articulaciones que soportan peso, como las rodillas o los tobillos; la mayoría de los pacientes reportan una sensación de parestesia cálida antes del sangrado articular que 


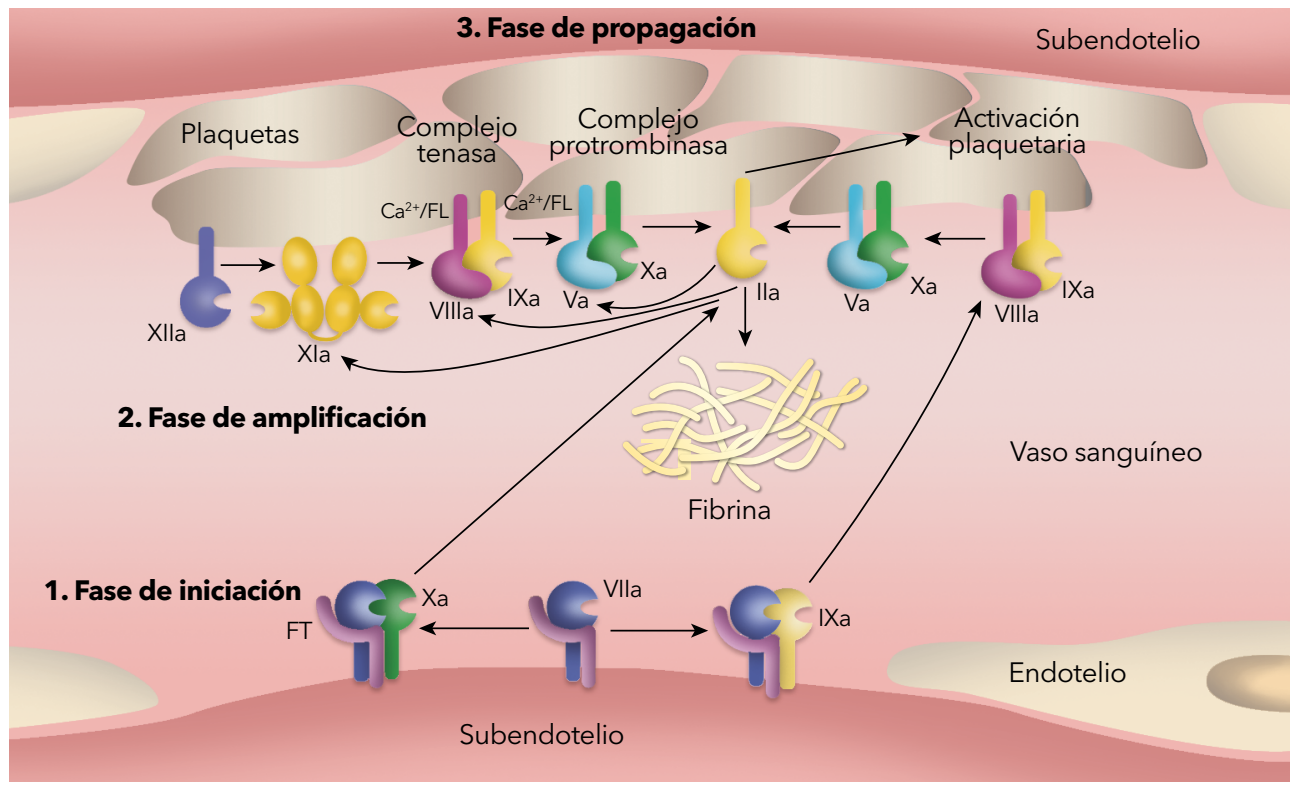

Figura 4. Modelo celular de la cascada de la coagulación. 1. Fase de iniciación: el complejo FT/VIla activa los factores X y IX para formar pequeñas cantidades de trombina (factor lla); 2. Fase de amplificación: la trombina formada activa los factores XI, VIII y $\vee$ para que sirvan de cofactores y así estimular una mayor producción de trombina, que a su vez acelera la activación plaquetaria para la unión de factores de coagulación; 3. Fase de propagación: el factor Xla activa al factor IX para formar complejo tenasa (IXa/VIIla/ $\mathrm{Ca}^{2+} / \mathrm{FL}$ ), que participa en la activación de mayores cantidades de factor $X$ que forma el complejo protrombinasa $\left(\mathrm{Xa} / \mathrm{Va} / \mathrm{Ca}^{2+} / \mathrm{FL}\right)$, y cataliza la formación de trombina suficiente para producir fibrina. $\mathrm{FT}$ : factor tisular; $\mathrm{Ca}^{2+}$ : calcio; FL: fosfolípidos.

se le conoce como "aura" [28]. También se presentan hematomas subcutáneos o musculares profundos, como el del psoas, que pueden simular una apendicitis. Las manifestaciones hemorrágicas pueden afectar cualquier parte del cuerpo en forma de sangrado ocular, renal (hematuria) o digestivo; no es raro observar hematemesis o melena por sangrado gastrointestinal superior, relacionado con úlceras pépticas o gastritis. Es común la epistaxis, hemorragia de la boca, las encías, los labios, el frenillo y la lengua; otras manifestaciones pueden ser metrorragias y hemorragias cerebrales $[28,29]$. Las formas leves suelen ser clínicamente silenciosas hasta que un evento traumático lo pone de manifiesto. Por lo general, la primera manifesta- ción de sangrado se presenta en niños que son llevados a procedimientos quirúrgicos $[3,5]$.

\section{Clasificación}

La hemofilia $B$, de acuerdo a la actividad de coagulación del factor IX, se puede clasificar de la siguiente manera:

- Severa, cuando no hay detección del factor $(0,01 \mathrm{UI} / \mathrm{mL} 0<1 \%)$. Estos pacientes cursan con sangrado espontáneo en las articulaciones o músculos.

- Moderada (0,01 UI/mL a 0,05 UI/ $\mathrm{mL}$, o de $1 \%$ a $5 \%$ ). Estos pacientes 
pueden presentar sangrado espontáneo ocasional o sangrado prolongado con traumas o cirugías menores.

- Leve $(0,05 \mathrm{UI} / \mathrm{mL}$ a 0,4 UI/mL, o de $5 \%$ a $40 \%$ ). Se manifiesta como sangrado severo con traumas o cirugías mayores. El sangrado espontáneo en estos pacientes es raro [27,30-32].

Debido a que solo el $2 \%$ de los individuos afectados tienen niveles entre $40 \%$ y $50 \%$, su clasificación es ambigua y sigue sin resolverse [31,33]; la mayoría de ellos se ubican en la de grado leve [33].

\section{Diagnóstico}

Las manifestaciones hemorrágicas sin causa u origen traumático y los sangrados prolongados después de procedimientos quirúrgicos, requieren de valoración del sistema hemostático con hemograma, tiempo de tromboplastina parcial activado (TPT), tiempo de protrombina (TP), tiempo de trombina (TT), PFA-200 y factores de la coagulación, para hacer el diagnóstico diferencial con patologías como la hemofilia A, entre otras [34,35]. La historia familiar del paciente es un dato muy importante en estos casos, además de la historia clínica de hemorragia que suele ocurrir durante la infancia cuando se inicia el gateo [36]. A continuación se describen las pruebas de coagulación de mayor utilidad para el diagnóstico de hemofilia $B$, con base en las pruebas disponibles que evalúan la cascada clásica de la coagulación.

\section{Tiempo de tromboplastina parcial activado (TPT)}

Evalúa las vías intrínseca y común de la cascada de coagulación, específicamen- te los factores II, V, VIII, IX, X y XI, y otros factores de contacto como el XII, la precalicreína y el QAPM [37]. Esta prueba es muy útil en el tamizaje de deficiencias de factores de la coagulación heredados, como la hemofilia $B$, y en los adquiridos, como sucede cuando hay presencia de un inhibidor. La prueba se realiza agregando el reactivo comercial que contiene una mezcla de tromboplastinas como la cefalina o la fosfatida, con un activador de contacto como el caolín, la sílica micronizada o el ácido elágico, y un substituto de los fosfolípidos de las plaquetas para eliminar la variabilidad inducida por la alteración del número o función plaquetaria. A continuación, el plasma citratado del paciente se incuba por varios minutos y se le agrega clorhidrato de calcio; finalmente, se calcula el tiempo de formación del coágulo tomando en cuenta el tipo de reactivo usado, el tipo de anticoagulante y la técnica instrumental utilizada [18]. El valor normal de TPT varía según los reactivos y los analizadores, pero su rango puede estar aproximadamente entre 26,8 a 34,5 segundos, y se puede alargar en casos de deficiencias, disfunción o inhibición de los factores XII, XI, IX, VIII, X, V, II, fibrinógeno, precalicreína y QAPM, contaminación por heparina, anticuerpos antifosfolípídos, presencia de anticoagulante lúpico y productos de degradación de la fibrina elevados [18].

Se puede dar una falsa prolongación del TPT en el caso de pacientes con procesos inflamatorios que presentan proteína $C$ reactiva (PCR) aumentada, ya que la PCR es una proteína de fase aguda con afinidad por los fosfolípidos, y estos son utilizados como una superficie catalítica en las pruebas de TPT [38]. En la hemofilia $B$, el TPT se encuentra usualmente alargado, aunque ocasionalmente en la forma leve, puede estar normal o mínimamente prolongado [39], por 
lo que en un paciente con historia clínica y familiar hemorrágica, a pesar de que esta prueba esté normal, debe medirse la actividad de los factores de coagulación, tomando en cuenta que el análisis genético molecular es el que puede confirmar el diagnóstico y revelar correlaciones genotipofenotipo [39].

\section{Tiempo de protrombina (TP)}

Esta prueba se usa para evaluar la función de las vías extrínseca y común de la cascada de coagulación, específicamente los factores II, V, VII y X. Su valor normal varía según los reactivos y los analizadores, pero su rango puede estar aproximadamente entre 9,7 a 11,8 segundos, y se puede prolongar en casos de concentraciones elevadas de heparina y anticuerpos antifosfolípidos, en presencia de productos de degradación de la fibrina [17], en enfermedad crónica del hígado, y cuando hay deficiencia leve de vitamina $\mathrm{K}$ por tratamiento con antagonistas de la vitamina K [40]. No detecta deficiencia del factor IX, por lo que debería ser normal en pacientes con sospecha de hemofilia B [37].

\section{Tiempo de trombina (TT) y fibrinógeno}

El tiempo de trombina debe incluirse en el estudio de coagulopatías de tipo hemorrágico. Su valor de referencia es de 13,4 a 21 segundos. Evalúa la actividad del fibrinógeno en el proceso hemostático para convertirse en hebras de fibrina. Suele estar prolongado en caso de deficiencia, ausencia total o disfunción del fibrinógeno, por lo que en estos casos debería evaluarse el nivel de fibrinógeno, cuyo valor normal es de 200 a 400 mg/dL. El TT también se prolonga ante la presencia de productos de degradación del fibrinógeno, inhibidores directos de trombina, terapias con ácido valproico y paraproteínas [37]. Así como el TP, este también suele ser normal en hemofilia $B$.

\section{Mezcla o prueba de corrección}

Se puede realizar una prueba de mezcla o de corrección en caso de un TPT prolongado para diferenciar entre la deficiencia de un factor de coagulación y la presencia de heparina o de un inhibidor. Para ello, se mezcla una cantidad de plasma del paciente con un pool de plasma normal en proporciones iguales, y se repite la determinación del TPT. La normalización del TPT después de la prueba indica una deficiencia de factores de la coagulación; por el contrario, la persistencia del TPT prolongado es indicativo de la presencia de heparina, de un inhibidor específico de los factores de la coagulación, principalmente del factor VIII O del factor IX (hemofilias adquiridas), o de anticoagulante lúpico $[11,18,41]$.

\section{PFA-200}

El PFA-200 simula in vitro las condiciones hemodinámicas de la adhesión y agregación de las plaquetas. Ante la presencia de manifestaciones hemorrágicas que reflejen trastornos plaquetarios o de vasos sanguíneos, tales como petequias, equimosis superficiales, epistaxis o sangrado profuso por heridas pequeñas, y en pacientes mayores con sangrado gastrointestinal [42], el PFA-200 es una de las pruebas de tamización de trastornos funcionales de las plaquetas, trombocitopenias y enfermedad de von Willebrand, que evalúa la hemostasia primaria y sustituye el tiempo de san- 
gría; además, es una herramienta de importancia en la evaluación de pacientes con tratamiento antiagregante [43]. El valor normal del PFA-200 colágeno/epinefrina es de 80 a 150 segundos, y con el sustrato colágeno/ ADP es de 60 a 100 segundos [44]. Se puede alargar en enfermedades hepáticas o renales y no tiene valor pronóstico de sangrado [40]. No es útil para el diagnóstico de deficiencia de factores de la coagulación, incluido el factor IX; sin embargo, ayuda a hacer un diagnóstico diferencial en caso de sangrado intraarticular en los subtipos más severos de enfermedad de von Willebrand tipo $2 \mathrm{~N}$ o tipo 3 que se asocian con disminución del factor VIII $[42,45,46]$, o en pacientes hemofílicos leves o moderados que solo presenten epistaxis o hemorragia de la boca, las encías, los labios, el frenillo y la lengua, y sangrados con heridas leves o posquirúrgicos [28], en los que esta prueba resulta normal.

\section{Nivel funcional de los factores de la coagulación}

El paso a seguir ante la presencia de manifestaciones hemorrágicas con un TPT alargado, sin otras pruebas alteradas, y que corrige con plasma normal, es la determinación de los niveles funcionales de los factores de la coagulación. Los niveles funcionales se pueden evaluar por dos métodos; uno por medición coagulométrica que determina los factores de la vía intrínseca y común, utilizando la prueba de TPT, y el otro método, por medición cromogénica de la actividad de los factores VIII o IX en plasma. En la medición coagulométrica se realizan tres diluciones del plasma del paciente con un volumen igual de plasma deficiente del factor que se quiere estudiar, y se realiza un TPT para cada dilución. El tiempo que se requiere para la formación del coágulo dependerá del nivel del factor presente en la muestra del paciente [47].

Por su parte, la prueba cromogénica se realiza en dos etapas; en la primera, el plasma del paciente se mezcla con los reactivos y sustratos apropiados que inducen la activación rápida de factor $X$. La cantidad de factor Xa formado será proporcional al nivel de factor funcional que se quiere estudiar en la muestra del paciente. En la segunda etapa de la prueba, se adiciona un sustrato cromogénico específico para el factor $\mathrm{Xa}$, y se cuantifica su concentración fotométricamente; la intensidad del color será proporcional al nivel del factor en estudio, en este caso el factor IX [47].

La medición coagulométrica a pesar de ser la más utilizada, puede estar asociada con limitaciones en la detección de niveles reducidos de factor IX en pacientes con hemofilia B leve; sin embargo, es rápida, de bajo costo y fácil de automatizar. La prueba cromogénica es más lenta, no es últil para un análisis de emergencia, y faltan protocolos estandarizados para su aplicación en algunos laboratorios; no obstante, esta se realiza típicamente con una dilución en lugar de tres, y no requiere la adición de plasma deficiente en factor, lo que ahorra costos. Además, es más resistente a los interferentes de los anticoagulantes lúpicos y anticoagulantes orales [47]. Actualmente no hay datos suficientes para sugerir que solo se deba realizar la prueba de actividad cromogénica en la evaluación de la hemofilia B leve, y usualmente se sugiere el uso combinado de ambas pruebas $[47,48]$. También se recomienda el uso de ambas pruebas en el seguimiento de los pacientes con hemofilia B para 
determinar la cinética del factor IX posterior a la administración de los concentrados del mismo, ya que existen estudios que han demostrado un fenómeno de pérdida progresiva de la actividad biológica del factor IX después de su infusión [49].

En caso de obtenerse valores deficientes de factores dependientes de vitamina $K(X, I X, V I I, I I)$, es indispensable descartar primero la deficiencia de esta vitamina o el uso de antagonistas como la warfarina [15]. Otra situación en la que pueden estar disminuidos es en niños hasta los 6 meses de edad, ya que los niveles de la mayoría de los factores de coagulación son aproximadamente el $50 \%$ de los niveles del adulto, y se reducen aún más en prematuros infantes al $30 \%$ entre las semanas 24 a 29 de gestación, y al $20 \%$ entre las semanas 19 a $23[50,51]$.

\section{Tromboelastografía}

La tromboelastografía (TEG) es utilizada para evaluar la función de la coagulación integral. La técnica mide y muestra gráficamente los cambios en la viscoelasticidad, en tiempo real, de todas las fases del desarrollo y resolución de los coágulos [52]; a pesar de que fue descrita por primera vez hace más de 70 años en 1948, ha demostrado su utilidad clínica solo durante los últimos $30[48,49]$. Permite documentar gráficamente las distintas etapas de la coagulación: la formación de fibrina, la retracción del coágulo, la agregación plaquetaria y la lisis del coágulo.

Esta técnica es ideal en los casos de hemofilia B cuando existen discrepancias entre el fenotipo de laboratorio (severa, moderada o leve) y la gravedad de las manifestaciones clínicas, y para una mejor comprensión de la heterogeneidad en la hemostasia de estos pacientes, la cual también puede tener un origen multifactorial [52]. Asimismo, tiene utilidad en la planificación de una profilaxis adecuada a largo plazo, y en el monitoreo de las nuevas opciones terapéuticas en hemofilia B [52].

\section{Estudios moleculares}

El diagnóstico de hemofilia $B$ se establece por el nivel de actividad del factor IX, pero se puede confirmar por medio de un estudio molecular en los casos hereditarios [3,5]. La prueba de secuenciación es actualmente el método de elección para la caracterización de las mutaciones en el gen F9, la cual permite una visualización directa de su localización y el tipo de rearreglo genético involucrado; este es el método diagnóstico de mayor precisión para la detección de portadoras, con una confiabilidad mayor del $99 \%$, y es la herramienta básica para el conocimiento de la etiología a nivel molecular que posee la enfermedad [4]; asimismo, es de utilidad para predecir el desarrollo de inhibidores [14]. La detección de mutaciones para el asesoramiento familiar se incorpora cada vez más a la atención clínica de rutina [53].

Finalmente, es importante tener presente que debido a que existen trastornos de coagulación que comparten manifestaciones hemorrágicas similares, es fundamental realizar un diagnóstico correcto con el soporte de un servicio de laboratorio completo de pruebas coagulación, con el fin de que el paciente reciba el tratamiento apropiado [27]. En la figura 5 se observa un algoritmo para el diagnóstico de hemofilia B. 


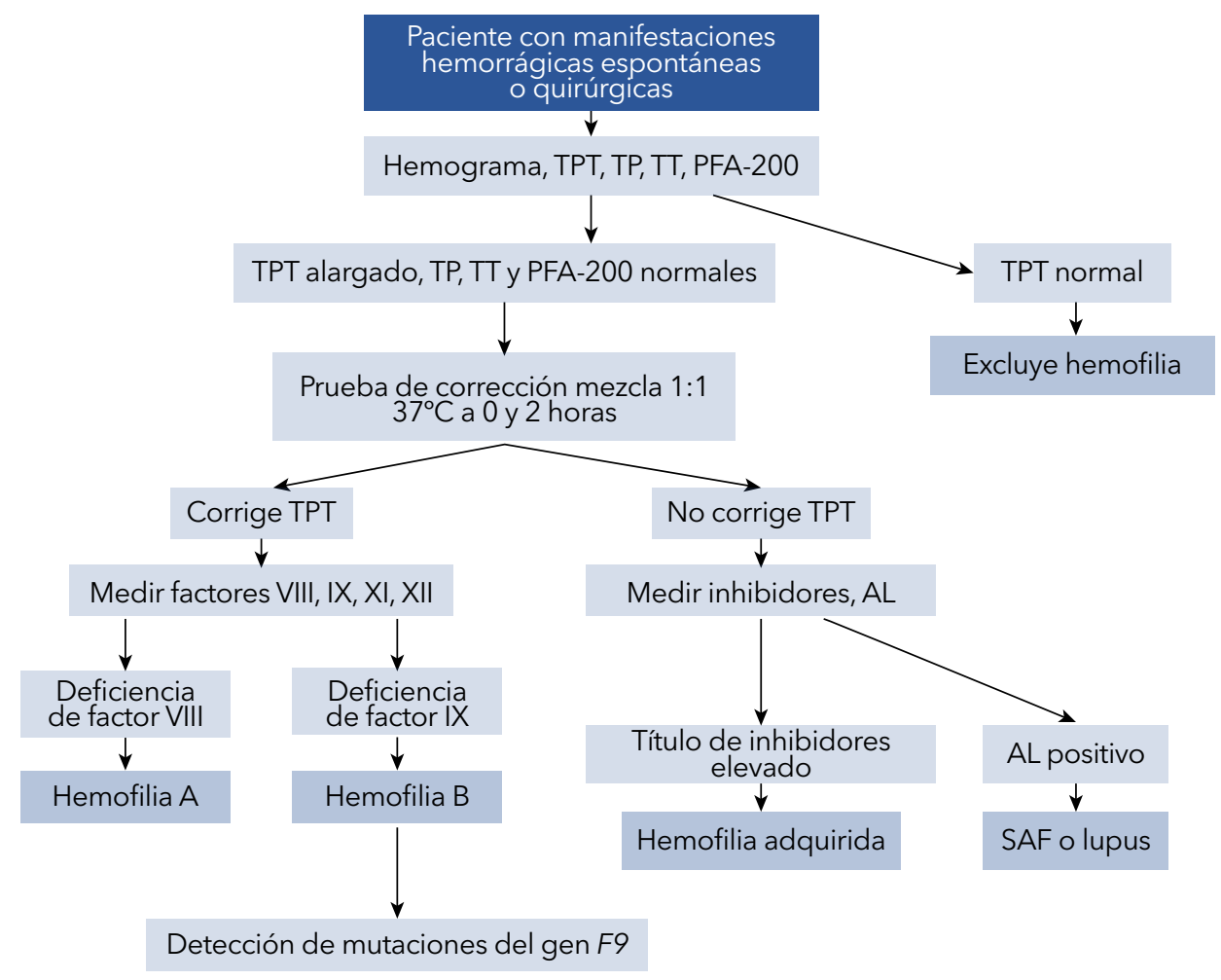

Figura 5. Algoritmo para el diagnóstico de hemofilia B. TPT: tiempo de tromboplastina parcial activado; TP: tiempo de protrombina; TT: tiempo de trombina; AL: anticoagulante lúpico; SAF: síndrome antifosfolípido.

\section{Tratamiento}

En la década de los 80 , miles de personas fallecieron a causa del tratamiento para la hemofilia debido a que se utilizaron concentrados de factores de coagulación fabricados a partir de plasmas de donantes, que facilitaron la transmisión del virus de la inmunodeficiencia huma$\mathrm{na}(\mathrm{VIH})$ y del virus de la hepatitis $\mathrm{C}(\mathrm{VHC})$ [54]; posteriormente, las técnicas de inactivación viral y los nuevos métodos de detección de virus en las donaciones de sangre, mejoraron la seguridad de los productos derivados del plasma. Finalmente, los avances de la tecnología de ADN han permitido la producción industrial de los concentrados de factor
IX recombinante que son utilizados en la actualidad [54]. La cantidad del factor IX infundido depende de la severidad del sangrado, el sitio en el que ocurrió la hemorragia y las medidas antropométricas del paciente $[10,19,29,30]$. Una unidad infundida de concentrado de factor IX por $\mathrm{kg}$ de peso incrementa el nivel del factor en $0,8 \mathrm{UI} / \mathrm{dL}$ en adultos y en 0,7 $\mathrm{UI} / \mathrm{dL}$ en niños [27]; el aumento deseado del factor IX es usualmente $40 \%$ para hemorragias moderadas, $50 \%$ para hemorragias severas, y entre $80 \%$ y $100 \%$ para hemorragias potencialmente mortales [27].

En los últimos años han surgido nuevas opciones de tratamiento como 
la terapia génica con vectores virales adeno-asociados ( $A A V$, del inglés, Adeno-Associated Virus) o con lentivirus recombinantes. Los virus que contienen los genes terapéuticos infectan las células del paciente para que se produzca factor IX circulante a niveles fácilmente detectables, restaurando de forma permanente los niveles del factor de coagulación en la circulación $[30,55,56]$. Aunque aún se encuentran en etapa de investigación, los resultados son prometedores [57]; sin embargo, esta terapia no es aplicable para todos los pacientes, ya que hasta el $50 \%$ de la población tiene anticuerpos neutralizantes contra los AAV [58], y es una terapia con alto costo para países como el nuestro.

Otras terapias basadas en modelos animales son el uso de células madres cultivadas para diferenciarse en células funcionales similares a los hepatocitos, las cuales demostraron mejorar la actividad del factor IX, la hemostasia y la generación de trombina, cuando fueron implantadas en ratones con hemofilia $B$ [59], y el trasplante de células madres mesenquimales de médula ósea, entre otras [58].

\section{Inhibidores}

Los inhibidores son anticuerpos policlonales (principalmente de tipo lgG) de alta afinidad que inhiben la función de los factores de coagulación [60], y pueden surgir como aloanticuerpos en pacientes que han recibido transfusiones de factor IX exógeno [61], los cuales tienen especificidad contra los dominios Gla y serina proteasa del factor IX [62]. La prevalencia del desarrollo de inhibidores en pacientes con hemofilia $B$ es menor que en pacientes con hemofilia $A$; en hemofilia $B$ seve- ra es de aproximadamente $1,5 \%$ a $3 \%$ [60], y en hemofilia B moderada y leve es extremadamente raro el desarrollo de inhibidores [62]. Existen varios factores predisponentes para el desarrollo de inhibidores, como son el tipo de mutación genética involucrada $[60,63]$, la edad del paciente y el número de infusiones de factores (en las primeras 50 infusiones existe mayor riesgo) [40]. Debe sospecharse un inhibidor si un paciente tiene mal control del sangrado con el uso de factores o si tiene niveles de factores significativamente más bajos de lo previsto, posteriores a la infusión. El desarrollo de inhibidores en hemofilia $B$ tiene una característica particular, y es que en el $60 \%$ de los casos, aproximadamente, va precedido de una reacción anafiláctica severa como respuesta a la terapia de reemplazo con factor IX $[11,63]$.

\section{Profilaxis}

Los regímenes de tratamiento profiláctico se dividen en profilaxis primaria, que consiste en la infusión regular del factor deficiente por más de 45 semanas/año (antes del inicio del daño articular), y en profilaxis secundaria, que consiste en la administración del factor deficiente, pero en presencia de daño articular [64].

Inicialmente se administraba el factor IX deficiente en los pacientes solo cuando iniciaban las manifestaciones hemorrágicas, pero en la actualidad esto ha cambiado y se le ha dado prioridad al tratamiento profiláctico al considerase el estándar de oro en la prevención de la artropatía hemofílica [65]. Asimismo, la profilaxis ha demostrado ser muy útil en la prevención de eventos que amenazan la vida, como las hemorragias intracraneales [64]. 


\section{Conclusiones}

Para el estudio de la hemofilia B se deben implementar herramientas de enfoque diagnóstico a través de pruebas organizadas como algoritmos, ya que no todos los trastornos hemorrágicos tienen una presentación clínica característica y muchas veces se omiten detalles o pruebas específicas que demoran el diagnóstico y las medidas terapéuticas adecuadas y oportunas.

La hemofilia representa una limitante para los afectados en todos los aspectos de la vida: biológicos, psicológicos y sociales. La expectativa de vida de pacientes con hemofilia $B$ depende de la severidad de la deficiencia del factor IX, de las medidas profilácticas y del tratamiento recibido. En la hemofilia leve y moderada esta expectativa es cercana a la de la población normal (75 años versus 78 años) [66]; sin embargo, en la hemofilia severa disminuye considerablemente a 63 años, ya que en estos casos cuando se desencadena un episodio hemorrágico puede ser una entidad potencialmente mortal [67]. La profilaxis juega un papel fundamental en la mejoría de la calidad de vida de los pacientes hemofílicos, en particular en los casos de enfermedad moderada y severa [68].

Por último, es de suma importancia educar a los individuos afectados para que tengan una visión más realista de su condición, y desde pequeños fomentarles las normas profilácticas y de tratamiento que ameriten; asimismo, se les deben proporcionar actualizaciones sobre los avances clínicos, especialmente en la medida en que los tratamientos se vayan adaptando a la condición de cada individuo. También se deben educar las familias de los individuos afectados, a través del asesoramiento genético [68].

\section{Referencias}

1. Shavit JA, Ginsburg D. Chapter 73. Hemophilias and other disorders of hemostasis. In: Rimoin D, Pyeritz R, Korf B, eds. Emery and Rimoin's Principles and Practice of Medical Genetics. Oxford: Academic Press; 2013. p. 1-33. https://doi.org/https://doi.org/10.1016/B9780-12-383834-6.00077-X.

2. Schulman S. Hemofilia leve. Edición revisada. In: Mahlangu J, ed. Tratamiento de la hemofilia $N^{\circ}$ 41. Montreal: Federación Mundial de Hemofilia (FMH); 2012. p. 1-13. Disponible en: http:// www1.wfh.org/publication/files/pdf-1193.pdf.

3. Philip J, Sarkar RS, Kumar S, Prathip BR, Pathak A. Factor IX deficiency (Christmas disease). Med J Armed Forces India 2012;68:379-380 https://doi.org/10.1016/j.mjafi.2011.12.007.

4. Goodeve AC. Hemophilia B: molecular pathogenesis and mutation analysis. J Thromb Haemost 2015;13:1184-1195. https://doi. org/10.1111/jth.12958.

5. García-Chávez J, Majluf-Cruz A. Hemofilia. Gac Med Mex 2013;149:308-321.

6. Kasper CK, Lin JC. Prevalence of sporadic and familial haemophilia. Haemophilia 2007;13:90-92. https://doi.org/10.1111/j.1365 2516.2006.01397.x.

7. Franchini M, Castaman G, Coppola A, Santoro C, Zanon E, Di Minno G, et al. Acquired inhibitors of clotting factors: AICE recommendations for diagnosis and management. Blood Transfus 2015;13:498-513. https://doi. org/10.2450/2015.0141-15.

8. Shetty S, Ghosh K, Mohanty D. Hemophilia B in a female. Acta Haematol 2001;106:115-117. https://doi.org/10.1159/000046599.

9. Staber J, Croteau SE, Davis J, Grabowski EF, Kouides P, Sidonio RF. The spectrum of bleeding in women and girls with haemophilia B. Haemophilia 2018;24:180-185. https://doi. org/10.1111/hae.13376.

10. García-Candel F. Estudio descriptivo mutacional de pacientes hemofílicos en la región de Murcia. Thesis. Medicina Interna, PhD. Murcia, España: Universidad de Murcia; 2013. p. 139.

11. Cugno M, Tedeschi A. Chapter 59. Coagulation factor autoantibodies. In: Shoenfeld Y, Meroni PL, Gershwin ME, eds. Autoantibodies. 3th ed. San 
Diego: Elsevier; 2014. p. 499-509. https://doi. org/10.1016/B978-0-444-56378-1.00059-9.

12. Monahan $\mathbf{P}$, Velander W, Bajaj P. Chapter 640 . Coagulation factor IXa. In: Barret A, Woessner F, Rawlings N, eds. Handbook of Proteolytic Enzymes. 3th ed. San Diego: Academic Press; 2013. p. 2898-2905. https://doi.org/10.1016/B978-012-382219-2.00640-2.

13. Brummel-Ziedins KE, Orfeo $T$, Everse $S J$, Mann KG. Blood coagulation and fibrinolysis. In: Greer JP, Arber DA, Glader BE, List AF, Means RM, Rodgers GM, eds. Wintrobe's Clinical Hematology. 14th ed. Philadelphia: Wolters Kluwer; 2019. p. 1466-1472.

14. Motlagh H, Pezeshkpoor B, Dorgalaleh A. Hemophilia B. In: Dorgalaleh A, ed. Congenital Bleeding Disorders. Cham, Suiza: Springer; 2018. p. 139-160.

15. Pruthi RK, Kluge ML, Ashrani AA. Coagulation disorders. In: Leonard DG, ed. Molecular Pathology in Clinical Practice. 2nd ed. Cham, Suiza: Springer; 2016. p. 203-223.

16. Escobar MA, Key NS. Chapter 123: Hemophilia A and hemophilia B. In: Kaushansky K, Prchal JT, Press OW, Lichtman MA, Levi M, Burns LJ, eds. Williams Hematology. 9th ed. New York: McGraw-Hill Education; 2015. p. 2126-2132.

17. Versteeg HH, Heemskerk JW, Levi M, Reitsma PH. New fundamentals in hemostasis. Physiol Rev 2013;93:327-358. https://doi.org/10.1152/ physrev.00016.2011.

18. Winter WE, Flax SD, Harris NS. Coagulation testing in the core laboratory. Lab Med 2017;48:295-313. https://doi.org/10.1093/lab$\mathrm{med} / \mathrm{lm} \times 050$.

19. Bermeo SM, Silva CT, Fonseca DJ, Restrepo CM. Hemofilia: diagnóstico molecular y alternativas de tratamiento. Colomb Medica 2007;38:308-315.

20. Lv X, Li T, Li H, Liu H, Wang Z, Guo ZP. Genetic analysis of a hemophilia $B$ family with a novel F9 gene mutation: A STROBE-compliant article. Medicine 2019;98:e15688. https://doi. org/10.1097/MD.0000000000015688.

21. Bowen DJ. Haemophilia $A$ and haemophilia B: molecular insights. Mol Pathol 2002;55:1-18. https://doi.org/10.1136/mp.55.1.1.

22. Gomez K, Chowdary P. Chapter 13. Hemophilia B: molecular basis. In: Lee C BE, Hoots
$W_{1}$ ed. Textbook of Hemophilia. New Jersey: Wiley-Blackwell; 2014. p. 97-102.

23. Smith SA. The cell-based model of coagulation. J Vet Emerg Crit Care 2009;19:3-10. https:// doi.org/10.1111/j.1476-4431.2009.00389.x.

24. Palta S, Saroa R, Palta A. Overview of the coagulation system. Indian J Anaesth 2014;58:515-523. https://doi.org/10.4103/0019-5049.144643.

25. McMichael M. New models of hemostasis. Top Companion Anim Med 2012;27:40-45. https:// doi.org/10.1053/j.tcam.2012.07.005.

26. Pérez-Gómez F, Bover R. La nueva cascada de la coagulación y su posible influencia en el difícil equilibrio entre trombosis y hemorragia. Rev Esp Cardiol 2007;60:1217-1219. https://doi. org/10.1157/13113924.

27. Srivastava A, Brewer AK, Mauser-Bunschoten EP, Key NS, Kitchen S, Llinas A, et al. Guidelines for the management of hemophilia. Haemophilia 2013;19:e1-e47. https://doi. org/10.1111/j.1365-2516.2012.02909.x.

28. Rodgers GM. Chapter 54. Inherited coagulation disorders. In: Greer JP, Arber DA, Glader BE, List AF, Means RM, Rodgers GM, eds. Wintrobe's Clinical Hematology. 14th ed. Philadelphia: Wolters Kluwer; 2019. p. 3653-3676.

29. Berges-García A, González-Ávila Al, Martínez-Murillo C, Gacía-Chávez J, Vélez-Ruela MA, Pompa-Garza MT, et al. Diagnóstico y tratamiento de hemofilia pediátrica. México: Secretaría de Salud; 2009. 1-52. 2012. Acceso 17 de noviembre de 2018. Disponible en http:// www.cenetec.salud.gob.mx/descargas/gpc/ CatalogoMaestro/141_GPC_HEMOFILIA_PEDIATRICA/Imss_ER.pdf.

30. Fijnvandraat $K$, Cnossen MH, Leebeek FWG, Peters M. Diagnosis and management of haemophilia. BMJ Brit Med J 2012;344:36-40. https://doi.org/10.1136/bmj.e2707.

31. Blanchette VS, Key NS, Ljung LR, Manco-Johnson MJ, van den Berg HM, Srivastava A, et al. Definitions in hemophilia: communication from the SSC of the ISTH. J Thromb Haemost 2014;12:1935-1939. https://doi.org/10.1111/ jth.12672.

32. Clausen N, Petrini $P$, Claeyssens-Donadel $S$, Gouw SC, Liesner R, PedNet and Research of Determinants of Inhibitor development Study Group. Similar bleeding phenotype 
in young children with haemophilia A or B: a cohort study. Haemophilia 2014;20:747-755. https://doi.org/10.1111/hae.12470.

33. Mazepa MA, Monahan PE, Baker JR, Riske BK, Soucie JM, U. S. Hemophilia Treatment Center Network. Men with severe hemophilia in the United States: birth cohort analysis of a large national database. Blood 2016;127:3073-3081. https:// doi.org/10.1182/blood-2015-10-675140.

34. Kruse-Jarres R, Kempton CL, Baudo F, Collins PW, Knoebl P, Leissinger CA, et al. Acquired hemophilia A: Updated review of evidence and treatment guidance. Am J Hematol 2017;92:695705. https://doi.org/10.1002/ajh.24777.

35. Jedidi I, Hdiji S, Ajmi N, Makni F, Masmoudi S, Elloumi M, et al. [Acquired haemophilia B: a case report and literature review]. Ann Biol Clin (Paris) 2011;69:685-688. https://doi.org/10.1684/ abc.2011.0638.

36. Tamagond SB, Hugar SI, Patil A, Huddar S. Christmas disease: diagnosis and management of a haemorrhagic diathesis following dentofacial trauma. BMJ case reports 2015;2015:bcr2014203790. https://doi. org/10.1136/bcr-2014-203790.

37. Hayward CP. How I investigate for bleeding disorders. Int J Lab Hematol 2018;40 Suppl 1:6-14. https://doi.org/10.1111/ijlh.12822.

38. van Rossum AP, Vlasveld LT, van den Hoven LJ, de Wit CW, Castel A. False prolongation of the activated partial thromboplastin time (aPTT) in inflammatory patients: interference of C-reactive protein. Br J Haematol 2012;157:394-395. https:// doi.org/10.1111/j.1365-2141.2011.08990.x.

39. Park CH, Seo JY, Kim HJ, Jang JH, Kim SH. A diagnostic challenge: mild hemophilia $B$ with normal activated partial thromboplastin time. Blood Coagul Fibrinolysis 2010;21:368-371. https://doi. org/10.1097/MBC.0b013e3283367946.

40. De Loughery TG. Tests of hemostasis and thrombosis. In: De Loughery TG, ed. Hemostasis and Thrombosis. 3rd ed. Cham, Suiza: Springer; 2015. p. 9-14.

41. van Herrewegen $F$, Meijers JC, Peters $M$, van Ommen CH. Clinical practice: the bleeding child. Part II: disorders of secondary hemostasis and fibrinolysis. Eur J Pediatr 2012;171:207-214. https://doi.org/10.1007/s00431-011-1571-x.
42. Leebeek FWG, Eikenboom JCJ. von Willebrand's disease. N Engl J Med 2016;375:2067-2080. https://doi.org/10.1056/ NEJMra1601561.

43. Campuzano-Maya G. PFA-100: una nueva prueba de función plaquetaria sustituta del tiempo de sangría. Med \& Lab 2013;19:511-548.

44. Rivillas-Rigol L, Vizcaíno-Carruyo JC, Tangarife-Castaño VJ, Campuzano-Zuluaga G, ToroMontoya Al. Pruebas de función plaquetaria por el sistema PFA-200. Med Lab 2020;24:162-164. https://doi.org/10.36384/01232576.215.

45. van Galen KPM, Mauser-Bunschoten EP, Leebeek FWG. Hemophilic arthropathy in patients with von Willebrand disease. Blood Rev 2012;26:261-266. https://doi.org/https://doi. org/10.1016/j.blre.2012.09.002.

46. Rodgers GM. von Willebrand disease-clinical manifestations. In: Greer JP, Arber DA, Glader BE, List AF, Means RM, Rodgers GM, eds. Wintrobe's Clinical Hematology. Philadelphia: Wolters Kluwe; 2019. p. 3676-3688.

47. Marlar RA, Strandberg K, Shima M, Adcock DM. Clinical utility and impact of the use of the chromogenic vs one-stage factor activity assays in haemophilia A and B. Eur J Haematol 2020;104:314. https://doi.org/10.1111/ejh.13339.

48. Peyvandi F, Oldenburg J, Friedman KD. A critical appraisal of one-stage and chromogenic assays of factor VIII activity. J Thromb Haemost 2016;14:248-261. https://doi.org/10.1111/ jth.13215.

49. Liebman HA, Rosenwald-Zuckerman T, Retzios A, Yasmin S, Kasper CK. Kinetics of factor IX activity differ from that of factor IX antigen in patients with haemophilia $B$ receiving high-purity factor IX replacement. Haemophilia 1999;5:174-180. https://doi.org/10.1046/j.13652516.1999.00305.x.

50. Pal S, Curley A, Stanworth SJ. Interpretation of clotting tests in the neonate. Arch Dis Child Fetal Neonatal Ed 2015;100:F270-274. https:// doi.org/10.1136/archdischild-2014-306196.

51. Pichler E, Pichler L. The neonatal coagulation system and the vitamin $\mathrm{K}$ deficiency bleeding: a mini review. Wien Med Wochenschr 2008;158:385-395. https://doi.org/10.1007/ s10354-008-0538-7. 
52. Nogami K. The utility of thromboelastography in inherited and acquired bleeding disorders. $\mathrm{Br} J$ Haematol 2016;174:503-514. https://doi. org/10.1111/bjh.14148.

53. Peyvandi F, Kunicki T, Lillicrap D. Genetic sequence analysis of inherited bleeding diseases. Blood 2013;122:3423-3431. https://doi. org/10.1182/blood-2013-05-505511.

54. Morfini M, Coppola A, Franchini M, Di Minno

G. Clinical use of factor VIII and factor IX concentrates. Blood Transfus 2013;11(Suppl 4):s55-s63. https://doi.org/10.2450/2013.010s.

55. Cancio MI, Reiss UM, Nathwani AC, Davidoff AM, Gray JT. Developments in the treatment of hemophilia B: focus on emerging gene therapy. Appl Clin Genet 2013;6:91-101. https://doi. org/10.2147/TACG.S31928.

56. George LA, Sullivan SK, Giermasz A, Rasko JE, Samelson-Jones BJ, Ducore J, et al. Hemophilia $B$ gene therapy with a high-specificactivity factor IX variant. New Engl J of Med 2017;377:2215-2227. https://doi.org/10.1056/ NEJMoa1708538.

57. VandenDriessche T, Chuah MK. Hemophilia gene therapy: ready for prime time? Hum Gene Ther 2017;28:1013-1023. https://doi. org/10.1089/hum.2017.116.

58. Dolan G, Benson G, Duffy A, Hermans C, Jiménez-Yuste V, Lambert $T$, et al. Haemophilia $\mathrm{B}$ : Where are we now and what does the future hold? Blood Rev 2018;32:52-60. https://doi. org/10.1016/j.blre.2017.08.007.

59. Wu YM, Huang YJ, Chen P, Hsu YC, Lin SW, Lai HS, et al. Hepatocyte-like cells derived from mouse induced pluripotent stem cells produce functional coagulation factor IX in a hemophilia B mouse model. Cell Transplant 2016;25:1237-1246. https://doi. org/10.3727/096368915×689541.

60. Santoro C, Quintavalle G, Castaman G, Baldacci E, Ferretti A, Riccardi F, et al. Inhibitors in He- mophilia B. Semin Thromb Hemost 2018;44:578589. https://doi.org/10.1055/s-0038-1660817.

61. Kasper CK. Diagnóstico y tratamiento de inhibidores de los factores VIII y IX. Discusión introductoria para médicos. In: Sam S, ed. Montreal, Canadá: Federación Mundial de Hemofilia; 2004. p. 1-13. Disponible en: http://www1.wfh.org/publications/files/pdf-1179.pdf.

62. Tandra A, Shapiro AD. Treatment of inhibitors in hemophilia B. In: Lee CA, Berntorp EE, Hoots WK, eds. Textbook of Hemophilia. 3rd ed. New Jersey: Wiley-Blackwell; 2014. p. 107-113. https://doi.org/10.1002/9781118398258.ch15.

63. Chitlur MB, Lusher JM. Chapter 14. Factor IX inhibitors in hemophilia B. In: Lee C, Berntorp E, Hoots W, eds. Textbook of Hemophilia. 3rd ed. New Jersey: Wiley-Blackwell; 2014. p. 103-106.

64. Moreno MM, Cuesta-Barriuso R. A history of prophylaxis in haemophilia. Blood Coagul Fibrinolysis 2019;30:55-57. https://doi.org/10.1097/ MBC. 0000000000000783 .

65. Makris M. Prophylaxis in haemophilia should be life-long. Blood Transfus 2012;10:165-168. https://doi.org/10.2450/2012.0147-11.

66. Thornburg CD, Duncan NA. Treatment adherence in hemophilia. Patient Prefer Adherence 2017;11:1677-1686. https://doi.org/10.2147/ PPA.S139851.

67. Darby SC, Kan SW, Spooner RJ, Giangrande PLF, Hill FGH, Hay CRM, et al. Mortality rates, life expectancy, and causes of death in people with hemophilia A or B in the United Kingdom who were not infected with HIV. Blood 2007;110:815-825. https://doi.org/10.1182/ blood-2006-10-050435.

68. Benson G, Auerswald G, Dolan G, Duffy A, Hermans $\mathbf{C}$, Ljung $\mathbf{R}$, et al. Diagnosis and care of patients with mild haemophilia: practical recommendations for clinical management. Blood Transfus 2018;16:535-544. https://doi. org/10.2450/2017.0150-17. 